\title{
Trivium
}

Revue franco-allemande de sciences humaines et sociales - Deutsch-französische Zeitschrift für Geistesund Sozialwissenschaften

31 | 2020

La culture politique de la République romaine

\section{Der Beruf des Bürgers. Vorwort}

\section{Claude Nicolet}

Traducteur : Andreas Wittenburg

\section{Q OpenEdition}

\section{Journals}

Édition électronique

URL : https://journals.openedition.org/trivium/7221

DOI : $10.4000 /$ trivium.7221

ISBN : 1963-1820

ISSN : $1963-1820$

\section{Éditeur}

Les éditions de la Maison des sciences de l'Homme

\section{Référence électronique}

Claude Nicolet, „Der Beruf des Bürgers. Vorwort", Trivium [Online], 31 | 2020, online erschienen am 30 Juni 2020, abgerufen am 24 August 2021. URL: http://journals.openedition.org/trivium/7221 ; DOI: https://doi.org/10.4000/trivium.7221

Ce document a été généré automatiquement le 24 août 2021.

\section{(c) () (9)}

Les contenus des la revue Trivium sont mis à disposition selon les termes de la Licence Creative Commons Attribution - Pas d'Utilisation Commerciale - Pas de Modification 4.0 International. 


\title{
Der Beruf des Bürgers. Vorwort
}

\author{
Claude Nicolet
}

Traduction : Andreas Wittenburg

\section{NOTE DE L'ÉDITEUR}

\section{Originalausgabe | édition originale}

»Avertissement «, in: C. Nicolet: Le métier de citoyen, Paris, Gallimard, 1976, S. 7-29.

Der Originaltext wurde leicht gekürzt und nicht alle Anmerkungen übernommen.

Auslassungen im Text sind durch [...] gekennzeichnet.

Wir danken den Rechtsnachfolgern, Herrn David Nicolet, Stéphane Nicolet und François Nicolet, für die freundliche Genehmigung, diesen Artikel in deutscher Übersetzung zu publizieren.

Nous remercions les ayants droit, MM. David Nicolet, Stéphane Nicolet et François Nicolet, de nous avoir accordé l'autorisation de traduire ce texte pour le présent numéro.

Dieses Buch ist in gewisser Weise die Geschichte eines Erstaunens und eines Bedauerns. Der Historiker, der sich mit Rom beschäftigt, und dabei insbesondere mit der Zeit der Römischen Republik, ist vor allem Historiker einer Oligarchie. Die großartige Römische Republik [...], diese machtvolle und die ganze Welt verschlingende Maschinerie, läuft Gefahr, sich für ihn aufgrund einer unvermeidlichen Sichtweise auf diese dünne Schicht von Beamten und Generälen, von Senatoren, Offizieren und Publikanen zu reduzieren. Es gilt, deren Rekrutierung zu untersuchen, ihre Machtbefugnisse $\mathrm{zu}$ umreißen, ihr Verhalten zu erforschen und, wenn möglich, ihre Handlungsweise zu erklären, und sei es auch nur, um so dem Gebot unserer Quellen zu folgen. Diese Personen in ihrer »ursprünglichen Schlichtheit« oder ihrer Maßlosigkeit von der Art der Helden Skakespeares sind es, die jene religiöse, militärische oder politische Chronik, die den wesentlichen Teil der historiographischen Überlieferung der Antike ausmacht, bevorzugt ans Licht gebracht hat. Fügen wir dem gerechterweise noch einige Helfer hinzu (Juristen, Redner, Dichter oder Gelehrte), denen jede Schriftkultur noch einen bevorzugten Platz einräumen würde: das Ergebnis ist spärlich und das Schauspiel 
bringt gegen alle Erwartungen nur eine lächerlich kleine Zahl von Darstellern auf die Bühne. Wie die meisten Historiker habe ich gezwungenermaßen der Untersuchung der offensichtlichen oder verborgenen Zeugnisse dieses Schauspiels zwanzig Jahre lang die weiterhin durchaus notwendigen Anstrengungen gewidmet. Die Untersuchung der römischen Oligarchie in ihren Strukturen wie in ihrer Entwicklung und in ihren regulären oder außerordentlichen Verhaltensweisen ist mehr als nur legitim - sie ist unerlässlich. Und vor allem ist sie alles andere als abgeschlossen. Es handelt sich doch am Ende, wie Ronald Syme sagt, um diejenigen, »die Geschichte machen «. ${ }^{1}$ Um den einzelnen Akteuren, den Familien, den Institutionen ihren Platz zuzuweisen und die chronologische Abfolge der Ereignisse festzustellen, bedarf es noch geduldiger und kollektiver Arbeit, und das wird für lange Zeit so bleiben.

Über diese Hauptdarsteller darf man aber nicht die Nebenfiguren vergessen, ohne die Erstere niemals »in ihrem Schicksal das Schicksal der Menschheit verkörpern« hätten können. Gegenüber der übrigen Welt hat sich Rom immer in der Form der untrennbaren Dreieinigkeit dargestellt: "die Beamten, der Senat und das Römische Volk«. Die Oligarchie führte sich - ob nun aufrichtig oder nicht - auf eine Masse von Bürgern zurück, die sie vorgab zu repräsentieren und deren Interessen zu vertreten sie behauptete. Ihre Generäle befehligten Heere, die aus Bürgern und nicht aus passiven Söldnern bestanden. Ihre Beamten schuldeten ihre Stellung im Prinzip »dem Wohlwollen des Volkes« (selbst wenn sie sie in der Praxis ihrem Namen, den Übereinkommen der Faktionen oder der Gunst der führenden Männer verdankten). Ihre Publikanen überwiesen die Erträge einer manchmal alles verschlingenden Steuererhebung an den "Schatz des Römischen Volks". Der aufrichtige oder vorgetäuschte Gehorsam, den kollektiven Interessen der cives zu dienen, dient bis weit in die Zeit der Bürgerkriege hinein als Leitmotiv oder Alibi. Innerhalb der Mauern der Stadt wird diese Masse der Bürger vielleicht unterdrückt und bevormundet [...], aber nach Außen wird sie von der Klasse ihrer Führer systematisch gerühmt und verteidigt. Der einfache Bürger ist dem Fremden gegenüber durch die Gesetze und Waffen Roms geschützt und wird wie ein souveräner Fürst respektiert. Ein Gesetz, das im Jahre 100 v. Chr. ein besonderes militärisches Kommando im Krieg gegen die Piraten einrichtet, ${ }^{2}$ bringt das in großartiger Unbefangenheit zum Ausdruck:

"Dass der Konsul an alle Völker, die in Freundschaft und Bündnis mit dem Römischen Volk stehen, Briefe schickt, in denen er ihnen befiehlt dafür zu sorgen, dass die Römischen Bürger, die Latiner und ihre italischen Bundesgenossen nicht nur in allen Städten und Inseln des Orients ohne Gefahr ihren Geschäften nachgehen können, sondern darüber hinaus auf allen Meeren in aller Sicherheit Schiffahrt betreiben können."

Es kann daher kein Zweifel bestehen, dass die Größe Roms in der Tat auf der - nur selten aufgekündigten - Anhängerschaft der Bürger beruhte, die der herrschenden Oligarchie nicht allein ihre Rechtfertigung verschaffte, sondern ihr auch ihre Soldaten, Steuerzahler und die Masse der Emigranten, Händler oder Kolonisten lieferte, die die Welt romanisieren sollten. Ein »Herrenvolk«.

Ein paradoxer Umstand ist, dass die Historiker meiner Meinung nach vor diesem Volk eigentlich zugleich Erstaunen wie Bedauern verspüren sollten. Der Grund des Erstaunens wäre folgender: in keinem Werk gibt es meiner Kenntnis nach eine zufriedenstellende Antwort auf die einfache Frage, was die erlebte und in gewisser Weise existenzielle tägliche Bedeutung des römischen Bürgerrechts war. Was bedeutete der Status als Bürger konkret (und warum übte er eigentlich eine offenbar so große 
Anziehungskraft auf diejenigen aus, die ihn nicht besaßen)? Und Gegenstand des Bedauerns wäre, dass sie, die Historiker, sich mit dem banalen Hinweis auf einige naive Klischees zufriedengegeben haben. Zunächst das Klischee der Größe: Gavius aus Compsa wird von Verres am Ufer der Meerenge von Messina gekreuzigt und ruft angesichts jener Küste Italiens, auf dessen Boden seine Hinrichtung nicht möglich gewesen wäre, »ich bin Römischer Bürger«. Der römische Legionär aus Afrika oder aus Gallien, »der Lastenträger des Marius«, trägt seine fünfunddreißig Kilo Ausrüstung, überquert zwischen zwei Schlachten den Canal de Fos und errichtet jeden Abend ein uneinnehmbares Lager. Da ist das Römische Volk, das den gesamten Senat am Tag nach der katastrophalen Niederlage von Cannae begleitet, um den geschlagenen Konsul zu empfangen und ihm zu danken, dass er »nicht an der Republik verzweifelt« sei. Und dann zeigen sie die Schattenseite: der Bürger ohne männliche Kraft, der jeden Tag ein Almosen von seinem reichen Beschützer erbettelt, seine Stimme bei den Wahlen an den Höchstbietenden verkauft, der seinen ach so wohlhabenden Nachbarn während der Proscriptionen anzeigt, der jeden Monat seine kostenlose Ration Getreide erhält. Das sind Schulbeispiele, die von einer sich wiederholenden Überlieferung vorgetragen werden, welche sich in erster Linie auf ein verächtliches Desinteresse an den täglichen Realitäten gründet. Wie soll man das kollektive Schicksal einer Stadt wie Rom verstehen, die sich in ständiger geographischer und demographischer Expansion befindet, wie soll man die Regeln des politischen Lebens beschreiben und erklären (jene "politische Grammatik«, die Christian Meier kürzlich beschrieben hat), wie die wesentlichen Probleme des soziopolitischen Zusammenhalts behandeln, die Verbindungen und Kommunikationskanäle der Macht, wenn man von den Bürgern an der Basis, sei es individuell oder gemeinschaftlich, eine so geringe Vorstellung hat? Das Bedauern, dass man den Mann auf der Straße zu sehr vernachlässigt hatte, war daher für mich keineswegs eine sentimentale oder doktrinäre Frage, sondern entsprach ganz einfach dem Bedürfnis, die der politischen Klasse gewidmeten Untersuchungen auszudehnen und die andere Seite der Medaille zu betrachten.

\section{»Politische Klasse« und Masse der Bürger}

4 Nachdem das Vorhaben einmal formuliert war, galt es, den genauen Gegenstand der Forschung und die geeignete Methode festzulegen. Ich habe zu Beginn eine Unterscheidung zwischen dem gefordert, was ich in Ermangelung einer besseren Definition die "politische Klasse« Roms genannt habe, und andererseits der Masse der Bürger. Letztere war es, deren Stellung ich definieren wollte, um ihre Rolle zu erfassen, die sich in einer Reihe von Bereichen daraus ergab. Doch man muss zuallererst Rechenschaft über diese Einteilung ablegen, die willkürlich erscheinen mag. Gibt es in Rom wirklich eine "politische Klasse«? Und wenn das so ist, wie unterscheidet sie sich von der Masse? Die Frage ist umso berechtigter, als im römischen Italien, wie in allen antiken Städten, die Gesamtheit der Bürger nur eine Minderheit der Gesamtbevölkerung darstellt: an ihrer Seite begegnet man Halbbürgern und ansässigen Fremden, aber auch »Bundesgenossen" oder "Untertanen«. Sie sind zwar alle frei, nehmen aber nicht an allen politischen oder den Römern vorbehaltenen bürgerlichen Aktivitäten teil. Und dann kommt natürlich noch die Masse der Unfreien hinzu, die vielleicht die doppelte Zahl der Bürger ausmacht. Von daher kann die im Verhältnis zur Gesamtbevölkerung relativ begrenzte Bürgerschaft als solche in gewisser Weise in ihrer Besonderheit als »die» politische Klasse an und für sich erscheinen, die in vieler 
Hinsicht privilegiert ist. Andererseits könnte man an der Berechtigung der Bezeichnung "politische Klasse« in der antiken Stadt griechischer Prägung Zweifel hegen, in der die außerordentlich vielseitigen politischen Aufgaben häufig durch das Los zugeteilt werden und jeder »abwechselnd befiehlt und gehorcht«. Es erscheint mir indes, dass für Rom die Bezeichnung (man könnte sehr wohl auch das Wort Oligarchie benutzen) in zweifacher Hinsicht berechtigt ist.

5 Ich bin [...] zu der Überzeugung gelangt, dass die Ausübung der militärischen und zivilen höheren Funktionen (die höheren Offiziere, Tribune, Präfekten und Beamte, beginnend mit den geringeren Funktionen wie denen der Triumviri capitales und monetales) in rechtlicher Hinsicht schon vom Beginn der Republik an einer Qualifikation beim Zensus unterworfen war. Das Gleiche gilt infolgedessen auch für den Eintritt in den Senat, denn im allgemeinen stellten die Zensoren oder ad hoc dazu bestimmten Beamten die Liste aus der Zahl der ehemaligen Beamten zusammen. Wenn es unter exzeptionellen Umständen dazu kam, dass man eine außerordentliche Ergänzung des Senats vornahm und man dabei auf Männer zurückgriff, die noch keinerlei Funktion ausgeübt hatten, mussten sie mindestens dem Ritterstand angehören. Der minimale Zensus, der für den Zugang zu den Magistraturen gefordert war, war im Übrigen die höhere Zensusklasse und damit genau die, die notwendig war, um in der Reiterschaft zu dienen. Man wird es mir erlassen, hier im Einzelnen all die Gründe aufzuführen, die mich in der letzten Zeit dazu gebracht haben, diese in der Tat neue These aufzustellen. Es mag genügen festzustellen, dass dieses Erfordernis eines minimalen Zensus, der dem des Ritterstandes entsprach, zunächst nur durch eine variable Zahl definiert war, von der wir lediglich wissen, dass sie wesentlich höher lag als die der Ersten Klasse der schwerbewaffneten Fußsoldaten. Gegen Beginn des 2. Jahrhunderts v. Chr. wurde diese Zahl auf Dauer (in einer neueingerichteten Währung) auf vierhunderttausend Sesterzen festgelegt, d.h. auf das Zehnfache der entsprechenden Zahl der Ersten Klasse. Senatoren und Ritter haben also dieselbe Qualifikation des Zensus bis in die Zeit des Augustus, der zu einem Zeitpunkt, den man auf die Jahre zwischen 18 und $13 \mathrm{v}$. Chr. datieren kann, den erforderlichen Zensus der Kandidaten für die Ämter und den Senat auf eine Million Sesterzen erhöhte und dabei Maßnahmen ergriff, die darüber hinaus die öffentlichen Funktionen und den Rang eines Senators praktisch erblich machten. Die römische Oligarchie ist also nicht nur eine faktische, die sich durch augenfällige soziologische Umstände von der Masse des Volkes abhebt, sondern eine rechtlich auf der Grundlage des Zensus festgelegte Oligarchie, also eine timokratische. Dieser Umstand muss uns nicht erstaunen, denn er ist auf die eine oder andere Weise allen antiken Städten gemeinsam: sie gründen sich alle auf ein System des Zensus, selbst die offensten und demokratischsten unter ihnen. Die Menschen der Antike waren stets der Meinung, dass gewisse Funktionen (und insbesondere die, bei denen es um die Verwaltung öffentlicher Mittel ging) allein von solchen Männern ausgeübt werden sollten, die Geld im Rücken hatten. Fast überall brachte die Erfüllung finanzieller Aufgaben die Hinterlegung von Garantien bei der Staatskasse mit sich. Ein elegantes Mittel zur Vereinfachung des Verfahrens war es, nur solche Kandidaten zuzulassen, die zu den obersten Klassen des Zensus gehörten. Rom galt bei Autoren, die wie Polybios oder Cicero Betrachtungen $\mathrm{zu}$ seiner Verfassung anstellten, niemals als eine demokratische Stadt, sondern bestenfalls als eine "gemilderte Oligarchie«. Wie man hinreichend sehen wird, war die Masse seiner Bürger durch den Zensus sorgfältig gegliedert und ihre ganz gewöhnlichen Aktivitäten wie die Teilnahme an den Versammlungen oder an den Wahlen hingen davon ab. Es wäre geradezu paradox 
gewesen, wenn die wichtigsten Aufgaben wie die Ausübung der Magistraturen von dieser Regel ausgenommen wären. Es gab also in der Stadt Rom infolge der ausdrücklichen Regeln der Verfassung eine einschneidende Trennung zwischen denen, die für eine direkte Beteiligung an der Politik qualifiziert waren, und den anderen. Das soll nicht heißen, dass es nicht doch gelegentlich Ausnahmen geben konnte. Man konnte zum Beispiel aufgrund des militärischen Verdienstes eine Ausnahme machen. Bei anderen Gelegenheiten, wie bei revolutionären Wirren oder bei Bürgerkriegen, konnten neue Männer, die diesmal aus den untersten Schichten kamen, sich aus der Masse hervorheben. Doch im Allgemeinen ging der Eintritt in die politische Klasse stets mit einem vorangehenden Erwerb von Reichtum einher. Der Zensus, das Vermögen, unterteilte also die Bürgerschaft immer entscheidend. Man muss jedoch sogleich eine wesentliche Einschränkung machen: in Rom beruhte die Hierarchie des Zensus niemals ausschließlich auf dem Vermögen. Sie gründete sich auch auf eine Reihe von anderen Gesichtspunkten, und darunter in erster Linie auf die Qualität und Reinheit des Erwerbs des Bürgerrechts: die Freigelassenen und selbst ihre Söhne waren im Allgemeinen (für eine oder zwei Generationen) bürgerlichen und politischen Beschränkungen unterworfen. Sie konnten individuell davon befreit werden durch eine Ausnahmeregelung, die im Prinzip in Anerkennung ihrer Verdienste getroffen wurde, auch wenn sie sie häufig einem einflussreichen Gönner verdankten. Wir finden hier zum ersten (aber nicht zum letzten) Mal diese kontinuierlichen Eingriffe in die soziale Hierarchie, die ihrer Natur nach unterschiedlich und in ständiger Bewegung sind, aber meist von den Bindungen des Klientelwesens und den bürgerlichen oder politischen Fähigkeiten bestimmt werden. Am Ende ist es in einer Gesellschaft dieser Art häufig vorteilhafter, Freigelassener oder ein anerkannter Sklave eines einflussreichen Schutzherren oder Besitzers zu sein als ein einfacher Bürger ohne Vermögen und ohne Einfluss. Daher sehen wir, wie sich im Umfeld der durch die Voraussetzungen des Zensus (und natürlich durch die effektive Ausübung der Funktionen) eindeutig definierten politischen Klasse ein größerer Kreis von Männern abzeichnet, der sich aus verschiedenen Gruppen zusammensetzt, aber deren Mitglieder alle mehr oder minder an bestimmten Formen der Macht teilhaben. Zunächst haben manche Funktionen in der Stadt der Antike einen ausgeprägteren politischen Charakter als in unseren Tagen. Zwei Kategorien heben sich in Rom vom Ende des 3. Jahrhunderts v. Chr. an deutlich hervor, und zwar die Funktionen der Rechtsprechung und die des Fiskus. Was Erstere betrifft, so waren die einfachen Bürger auf Dauer davon ausgeschlossen (im Gegensatz zu denen in Athen zum Beispiel). Vom Jahre $123 \mathrm{v}$. Chr. an werden sie durch einen irregulären, aber nicht aufzuhaltenden Prozess das Privileg und manchmal das Monopol der Ritter, die über den Zensus der Ritterklasse verfügen, aber nur unter der Bedingung, dass sie sich unter Beachtung einer impliziten Doktrin der "Gewaltenteilung " ${ }^{3}$, wie spätere Zeiten das bezeichnen würden, von den eigentlich politischen Funktionen fernhalten. Selbst in den Zivilprozessen unterliegen die Römer dem Urteil von Männern, die nicht nur die reichsten sind, sondern die in einer echten etablierten Körperschaft zusammengeschlossen sind (einem ordo), der vom Staat auf aktuellem Stand gehalten und genauestens überwacht wird. Das ist eine erstaunliche Zweiteilung. Die Funktionen des Fiskus bilden in unseren Augen ein noch erstaunlicheres Bild. Wie alle Städte und Staaten der Antike verfügte Rom für lange Zeit nicht über eine hinreichende Verwaltung und suchte in der Praxis die einfachsten Mittel, um die Einnahme fiskalischer oder anderer Mittel zu sichern, die es am Leben erhielten. Es hat daher systematisch und generell auf die Verpachtung der Eintreibung 
seiner Einkünfte zurückgegriffen. Das war eine passive, aber bequeme Vorgehensweise, die dem Staat ein regelmäßiges und von vornherein bekanntes Einkommen sicherte, wenn auch zum Nachteil der öffentlichen Kassen, wie man einräumen muss, und vor allem zum Nachteil der Steuerpflichtigen. Eine auf ein derartiges Verfahren der Eintreibung gegründete Fiskalität hat merkliche Auswirkungen auf das bürgerliche Verhalten. In Rom kommt noch ein besonderes Charakteristikum dazu: auch in diesem Fall waren die wichtigsten Steuerpächter, die sich in jeder Provinz zu dauerhaften "Gesellschaften« zusammenschlossen, notwendigerweise und verbindlich Ritter. Vom Zensus her gehörten sie also in dieselbe Klasse wie die Senatoren, und soziologisch gesehen stehen sich die beiden Gruppen sehr nah, aber von der Funktion her trennen sie tiefe und unvereinbare Gegensätze. Konflikte finanzieller, verwaltungsmäßiger und politischer Art scheiden sie voneinander. Der Ritterstand, der also die Richter und Publikanen stellt, erscheint wie eine Art zweiter »Adel«, ein privilegierter Stand mit starkem inneren Zusammenhalt, der in engem politischen und sozialen Kontakt mit dem senatorischen Milieu stand und ihm regelmäßig neue Männer als Nachschub lieferte. Zugleich bildet er eine politische oder, besser gesagt, führende Klasse, die sich indes, wenn auch sparsam, zur Basis hin, d. h. für einige Plebejer, öffnen kann.

Das Bild der römischen Führungsschicht wäre unvollständig, wenn man sich auf das bisherig Gesagte beschränken wollte. Man hat bis zum Überdruss wiederholt, dass die Republik keine wirkliche Verwaltung gekannt hat. Die mittleren und niederen Aufgaben, die unter der Verantwortung eines Beamten vollbracht wurden, seien einem aus Sklaven oder Freigelassenen bestehenden Personal anvertraut worden, das ihm oder zu ihm gehörte. Das ist zu Beginn ohne Frage zutreffend. Aber gegen Ende der Republik beginnt der Staat, eine Art von Reserve von untergeordneten Beamten zu schaffen, eine Art von »Liste der Tauglichen« (die immer noch als »Stände« bezeichnet werden), die so etwas wie den Ansatz einer Verwaltung darstellen. Diese Personen Pförtner, Schreiber usw. - sind in direktem Kontakt zu den Bürgern mit der Führung der Geschäfte beauftragt, sie verfügen in der Praxis über einen beträchtlichen Teil der Macht und haben vor allem Kontrolle über deren tatsächliche Ausübung. Ihre berufliche Kompetenz verschafft ihnen einen Vorteil gegenüber den häufig unerfahrenen Beamten, die Stetigkeit ihrer Stellung steht im Gegensatz zum raschen Wechsel der auf ein Jahr begrenzten Funktionen ihrer Herren. Für den Bürger sind sehr häufig sie es, die den Staat verkörpern.

Die Beamten und die Senatoren, die strenggenommen die politische Klasse Roms darstellen, werden, wie es normal ist in einer Gesellschaft, die zugleich auf Sklaverei, dem Patriarchat und dem Klientelwesen beruht, von ihrer unmittelbaren Umgebung unterstützt (und manchmal manipuliert), die sich aus Verwandten, Freunden, Klienten, Freigelassenen und Sklaven zusammensetzt. Diese Personen führen die täglichen Geschäfte für sie und verwalten insbesondere die Finanzen, kümmern sich um die technischen Aspekte ihrer Tätigkeit (indem sie zum Beispiel Reden, Briefe oder politische Texte verfassen) und sind häufig genug die wirklichen Urheber der Entscheidungen. Dort finden sich die engen und abenteuerlichen Wege, denen ein Plebejer folgt, wenn er in die politische Klasse aufsteigen will. 


\section{Rekrutierung und Verhaltensweisen}

8 Es geht hier nicht darum, diese politische Klasse zu untersuchen. In dieser Hinsicht sind die Bemühungen der heutigen Forschung bereits zu bemerkenswerten Ergebnissen gelangt. Vielleicht mit Ausnahme der ins Einzelne gehenden und vollständigen Untersuchung der Personen des Gefolges, die noch ansteht, kann man sagen, dass wir die römische Oligarchie inzwischen ziemlich gut kennen. Das gilt zunächst für ihre Zusammensetzung: Beamte, Senatoren, Ritter, Richter und Publikanen sind erfasst, identifiziert und in Hinblick auf ihren Ursprung und ihre Beziehungen untersucht worden. Diese Arbeit der Erfassung, die sich zum großen Teil auf die Methode der Prosopographie gründet, ist heute fast vollständig geleistet. Sie liefert uns eine hinreichend genaue und ins Einzelne gehende Betrachtung der führenden Schichten der Republik. Das Bild wird mit Sicherheit einige Korrekturen und Zusätze erfahren, aber es ist doch einigermaßen klar.

Wenn uns die Zusammensetzung ziemlich gut bekannt ist, sind indes die Verhaltensweisen dieser politischen Klasse nicht immer ebenso gründlich erforscht. Zweifellos sind die Gelehrten im Zeitraum von mehr als einem Jahrhundert dazu gelangt, den institutionellen Rahmen, in dem sich das politische Leben auf dieser gehobenen Ebene vollzieht, annähernd gründlich zu rekonstruieren: die Regeln der Übernahme und der Ausübung einer Magistratur, die Rolle und die Kompetenzen des Senats, das Kommando über die Provinzen oder das Funktionieren der Justiz sind in ihrer Theorie wie in ihrer Praxis zunehmend erforscht. Aber die Ergebnisse der prosopographischen Methode, auf die man seit Matthias Gelzer und Friedrich Münzer große Hoffnungen gesetzt hatte, sind doch vielleicht weniger gesichert, als man denkt. Wenn man fast das ganze politische Leben Roms mit dem Spiel der durch Heirat oder Familien geschaffenen Verbindungen, mit Verwandtschaft oder der Bildung von Parteien erklären will (das sind sicher wichtige Elemente, aber sie sind nicht die einzigen, die entscheidend sind), begibt man sich unvermeidlich in eine Sackgasse und macht sich allenfalls eine völlig empirische und zynische Vorstellung der Realitäten, die zu leichtfertig die Überzeugungen, die Moral, die rechtlichen Regeln sowie vor allem die Suche nach politischer Unterstützung und den Druck der öffentlichen Meinung, so schwach der auch sein mag, beiseiteschiebt. Aus diesem Grunde beginnen andere Methoden oder andere Gesichtspunkte, die in den meisten Fällen den Politischen Wissenschaften entlehnt sind, das Monopol der Prosopographie im rein politischen Bereich in Frage zu stellen. ${ }^{4}$ Statt von vornherein zu meinen, dass das Spiel der Clans und der Familien alles erklärt, sollte man sich zum Beispiel eher die Frage nach dem konkreten Verlauf der Entscheidungsprozesse stellen. Eine solche Untersuchung würde es erlauben, unterschiedliche Ebenen der Verantwortlichkeit zu bestimmen, und dazu unter anderem die Abfolge und das Niveau der nach Zeitpunkt und Umständen aufeinanderfolgenden Eingriffe oder die zur Blockierung eingesetzten Methoden zu erfassen. Indem man diese Wege der Betrachtung einschlägt, werden wir mit Sicherheit unsere Kenntnis der politischen Klasse Roms erneuern.

\section{Politisches und bürgerliches Leben}

Wie dem auch sei, das ist nicht mein unmittelbares Vorhaben. Da die Unterscheidung zwischen politischer Klasse und der Masse der Bürger in diesem Zusammenhang von 
Bedeutung ist, ist es Letztere und sie allein, die ich untersuchen wollte, ohne indes das stets gegenwärtige Problem der Kommunikation von der einen zur anderen Gruppe auf den Ebenen des Austauschs im personellen Bereich oder dem der Kommunikation zu meiden. Doch es ist angebracht, mein Vorhaben genauer zu fassen. Es kann sich nicht allein darum handeln, den Bereich zu erforschen, den man im Allgemeinen den politischen nennt, d.h. im Wesentlichen die Ausübung und die Kontrolle (sofern sie besteht) der Macht und der Fällung von Entscheidungen. Die römischen Institutionen sind, wie ich bereits gesagt habe, derart beschaffen, dass der Anteil der Masse der Bürger bei dieser Art von Aktivität sehr gering ist. Die einfachen Bürger, die weitgehend von den militärischen Kommandopositionen ausgeschlossen bleiben oder von den Magistraturen, ausgeschlossen auch von dem ständigen Rat der Stadt (dem Senat), sind doch mindestens Wähler, die dazu aufgerufen sind, ihre Stimme bei der Wahl der Beamten und dem Beschluss von Gesetzen abzugeben. Nun sind allerdings die Wählerstimmen, die aufgespalten und auf erstaunliche Weise hierarchisch gegliedert sind, nicht wirklich von Einfluss, außer denen der wohlhabendsten und vornehmsten unter den Bürgern. Außerdem sind die Regeln des Staatsrechts derart gestaltet, dass diese Abstimmungen die Form einer globalen Zustimmung oder Ablehnung haben müssen, und es scheint, dass die freie Information zwar nicht ausgeschlossen ist, aber doch zumindest die Erörterung und Debatte. Das ist wenigstens die allgemein anerkannte Lehrmeinung der Spezialisten des Römischen Staatsrechts. In jedem Falle riskiert man, nur wenig zu verstehen, wenn man nur das eigentlich politische Engagement der Bürgermassen in Rom berücksichtigt. Wir wollen einstweilen den ernüchternden und herablassenden Worten Ciceros Glauben schenken:

"Suche also nicht, Cato, die unteren Klassen der Genugtuung und des Interesses zu berauben, das sie darin finden, uns zu huldigen (uns den Hof zu machen, wenn wir Kandidaten sind). Lass denen, die alles von uns erwarten, das Mittel, dass auch sie uns etwas zukommen lassen können. Wenn das nur ihre eigene Stimme bei der Wahl ist, ist das doch wenig, denn angenommen, dass sie überhaupt Gelegenheit haben, sie uns zu geben, bleibt sie doch ohne jeden Einfluss. Und wie sie anerkennen, können sie sich weder für uns aussprechen, noch uns Bedingungen stellen, noch uns zu sich einladen [...].« (Cicero, Pro Murena 71).

Aber der politische Bereich ist nicht der einzige, in dem die Zugehörigkeit zur Bürgerschaft und der Status als Bürger entscheidende Folgen haben. Wo er Wähler war, war der römische Bürger auch und in erster Linie Soldat und zusätzlich noch Steuerzahler. Bei der weitgehenden Überzahl der politischen Auseinandersetzungen, die sich innerhalb der politischen Klasse vollzogen, aber an denen der Bürger als Wähler am Ende Anteil hatte, ging es um militärische oder finanzielle Fragen. Wie in jeder Stadt der Antike waren es die Leistungen, die nach einer in der Sprache der Bürger häufig verwendeten Formel »die Person und die Mittel« betrafen, die konkret die - günstige oder ungünstige - Position des Bürgers in der Gemeinschaft bestimmten. Das Leben als Bürger, das auf diese Weise für jeden von ihnen in der konkreten Anwendung als Gesamtheit der gemeinschaftlichen Rechte und Pflichten festgelegt war, übersteigt ziemlich weit den Bereich dessen, was im eigentlichen Sinne "politisch" zu nennen ist, denn häufig kann man davon ausgehen, dass diese Rechte oder Pflichten auf Entscheidungen zurückgingen, an denen das Volk keinen Anteil hatte. Es war daher angebracht, eine Bestandsaufnahme dieser Bereiche vorzunehmen, sie dabei zunächst voneinander $\mathrm{zu}$ unterscheiden und dann ihre gegenseitigen Beziehungen $\mathrm{zu}$ bestimmen. Das ursprüngliche Vorhaben weitete sich daher auf die Untersuchung eines 
Gesamtbildes aus, das, soweit möglich, sogar bis an die Grenzen des Bürgerrechts reichen sollte.

\section{Von der Regel zum Gelebten}

11 War das ein neues Vorhaben? Es wäre naiv und vermessen, das zu behaupten. Schließlich haben die Historiker des Römischen Privatrechts und des Römischen Staatsrechts seit Domenico Rubino und mit Sicherheit seit Rudolf von Ihering oder vor allem Theodor Mommsen bis zu Francesco De Martino unsere Kenntnis des rechtlichen Inhalts des Römischen Bürgerrechts unermüdlich vervollständigt. Großartige Rekonstruktionen bieten uns systematische und genaue Darstellungen, von denen jede ohne Frage von einer besonderen Sichtweise bestimmt und ohne Zweifel in gewissen Einzelheiten zu präzisieren ist, die aber doch einen dauerhaften Gewinn für die Wissenschaft darstellen. Von Anfang an will ich hervorheben, wie sehr ich diesen Giganten der Forschung verpflichtet bin. Dennoch ist folgendes zu sagen: wenn der Versuch, ein »Staatsrecht« des republikanischen Roms zu schreiben, wie es Mommsen getan hat, gewiss notwendig war; wenn man wie De Martino die Stufen seiner Entwicklung diachronisch verfolgen musste, indem man sie mit Sorgfalt in ihren politischen, wirtschaftlichen und sozialen Zusammenhang eingliederte; wenn man wie von Ihering versuchen konnte, aus diesen Regeln einen "Geist« des Privatrechts abzuleiten, neigt doch trotz allem die Sichtweise dieser Autoren, die allesamt von ihrer Ausbildung her Juristen sind, eher dazu, bewusst oder unbewusst der Regel Vorrang vor ihrer Anwendung einzuräumen, oder der Norm auf Kosten der Praxis, der Absicht des Gesetzgebers zum Nachteil des Gelebten und Konkreten. Das Römische Recht, vor allem das aus der Zeit der Republik, ist über die jahrhundertelang aufgebauten Schichten der Kodifizierung und der Kommentare der Kaiserzeit oder der byzantinischen Epoche auf uns gekommen. Diese Quellen neigen dazu, durch einen ständigen Einsatz der Logik die notwendigen Beziehungen der Personen und der Sachen wie der Regeln und der Konflikte in ein einheitliches System einzuordnen und in eine praktisch universale Sprache zu bringen, die es im wahrsten Sinne erlaubt, »Probleme« zu lösen. Das ist ein vielleicht einmaliges Phänomen in der Geschichte und führt zu einem faszinierenden Ergebnis. Dass es den Keim des gesamten Fortschritts im Bereich des modernen Rechts in sich trägt, ist wahrscheinlich. Doch es gibt keinen Aufschluss über die bescheidene tägliche Realität, besonders nicht die der Zeit der Republik. Bevor das Leben der römischen Bürger durch die Normen definiert und geordnet wurde, die die Juristen innerhalb der kaiserlichen Kanzlei oder den Schulen des Rechts sich bemühten, einsichtig zu machen, war es für sehr lange Zeit durch eine ganze Reihe aus der Gewohnheit entstandener Praktiken von sehr unterschiedlicher Bedeutung geregelt. Diese waren oft widersprüchlich oder offenbarten eine ganz archaische Logik oder einen archaischen Zusammenhang, der uns größtenteils unbekannt ist. Das ist noch der Zustand am Ende der Zeit der Republik. Im Übrigen hat dieses Bemühen um Logik und die Schaffung einer einsichtigen Sprache in Rom nie etwas anderes betroffen als das, was man Privatrecht nennen kann - was die Römer das ius civile nannten, das Recht der Bürger - und das ungefähr den Bereichen unseres Zivilrechts und unseres Strafrechts entspricht. Das Staatsrecht, d. h. das Recht, das die Beziehungen des Bürgers zum Staat sowie den speziellen Umgang der staatlichen Institutionen miteinander regelt, gehörte nicht eigentlich in die Kompetenz der Juristen und interessierte in der Tat wenig. Das war ein Bereich, den sie bereitwillig den 
Zufällen der Improvisation überließen, denn sie hielten es für außerordentlich wechselhaft. Wie sollte es daher erstaunlich sein, dass die Darstellungen, die man in der Moderne davon gegeben hat, die Systematik, die man darin zu finden versucht hat (z. B. Mommsen), kaum Ähnlichkeit mit der Realität haben? Um darzustellen, was von der Definition her kontingent, veränderlich und alltäglich ist, ist die Sprache des Rechts nicht gerade die geeignetste.

12 Auf der Suche nach diesem Alltäglichen und der Lebenserfahrung stößt man auf die erste Überraschung: derartige Praxisformen sind durch keinerlei juristische Regel festgelegt, d. h. sie haben ihren Ursprung in keinerlei Gesetz. Das ist etwa der Fall bei den Aufgaben, die aus der Pflicht zum Militärdienst erwuchsen, oder bei den steuerlichen Verpflichtungen, die die Lebensbedingungen der Bürger nicht weniger konkret bestimmten. Und wenn es Regeln des Staatsrechts gibt, so hat man stets Veranlassung, ihre reale Bedeutung zu bestimmen, d.h. die Ausnahmen, die sie zulassen, oder die Schwierigkeiten ihrer Anwendung. Vor allem wird man Sorge tragen, so genau wie möglich zu bewerten, was die Einhaltung dieser Verpflichtungen im Bereich der Lebensrealität für diejenigen bedeutet, die ihnen unterworfen sind, oder die Ausübung dieser Rechte für die, die sie genießen. Die Verpflichtung zum Wehrdienst bleibt selbst dann, wenn man ihre Regeln genau festlegt, ein abstrakter Sachverhalt, solange man nicht versucht, im Einzelnen zu erfassen, wer ihr unterlag und für wie lange, zu welchen Zeitpunkten im Jahr, in welchem geographischen Bereich sie zur Anwendung kam und in welchem Rhythmus; das gilt auch, solange man nicht versucht hat, für die Gemeinschaft wie für die Einzelnen, die dieser Verpflichtung unterworfen waren, eine demographische und finanzielle Bilanz zu ziehen; oder solange man nicht versucht hat, ein Bild von den Kommandostrukturen zu gewinnen, und zwar durch die "Werke und Tage« des römischen Legionärs zu einer bestimmten Epoche und an einem bestimmten Platz, vom Tag seines Einrückens bis zu dem seiner Entlassung, wenn er den denn erreichte. Und dieselbe Art von Untersuchung ist für die finanziellen Verpflichtungen notwendig oder für die Teilnahme an den Versammlungen. Das ist eine existentielle Geschichtsforschung, die dazu verurteilt ist, den realen Gehalt von Lebensbedingungen zu ergründen, deren juristischer Rahmen zwar besser bekannt ist, mich aber hier weniger interessierte. Vor allem ist das eine vielschichtige Geschichte. Es wäre eine große Gefahr und eine Quelle unverzeihlicher Irrtümer gewesen, den Status des Bürgers von Beginn an als einheitlich, gleichwertig und unabhängig von Unterschieden aller Art im physischen, ethnischen oder sozialen Bereich hinzustellen. Dass eine solche Abstraktion auf der Ebene der Rechtsgeschichte angebracht ist, ist zwar möglich, bleibt aber unter Beweis zu stellen. Auf der Ebene der Geschichte der Verhaltensweisen oder der Lebensführung, die ich umreißen wollte, wäre sie absurd gewesen. "Der « römische Bürger existiert nicht oder ist doch nur eine Schablone ohne Inhalt. Es gibt nur »die« römischen Bürger, die zugleich Landbesitzer und Produzenten sind, Römer aus Rom oder Bergbewohner aus dem Appenin, Nachfahren eines Konsuls oder neue Freigelassene. Dabei ist offensichtlich, dass diese Männer, so stark der Charakter des melting pot der römischen Bürgerschaft auch war oder sein wollte, ihr Bürgerrecht doch nicht auf dieselbe Weise ausübten. Diesen grundlegenden Fehler zu vermeiden ist mir allerdings sehr leichtgefallen, denn wir werden sehen, dass die Ausübung des Bürgerrechts in Rom doch eben gerade ungleich ist. Rechte und Pflichten sind ganz prinzipiell nicht identisch für jeden. ${ }^{5}$ Das Staatsrecht selbst forderte also von Anfang an einen Zugang zur Geschichte, der auf die 
Unterschiede achtete. Für mich ging es genau darum zu bestimmen, ob die Praxis diese Ungleichheit im Recht verstärkt oder im Gegenteil korrigiert.

\section{Eine erneuerte Lektüre}

13 Wenn sich so einigermaßen klar abzeichnete, welche Fragen man sich stellen sollte, blieb doch noch zu sehen, ob die Quellen es erlaubten, Antworten darauf zu geben. Mit anderen Worten, welche Methode stand für mein Vorhaben zur Verfügung? Der lange Umgang mit Mommsen war es [...], der mir eine seit langem geargwöhnte Antwort nahelegte: die antike Überlieferung, die auf uns gekommen ist, stellt zwar vor allem die Umstände und Taten der politischen Klasse in den Vordergrund, aber sie ist doch keineswegs stumm in Hinblick auf die konkreten Lebensumstände des einfachen Volks. Mommsen war sich dessen sehr wohl bewusst, wenn er als Gegengewicht zu den etwas dogmatischen Kapiteln, die er zu den Normen des Staatsrechts verfasste, die Seiten unten mit fast ausschweifenden Anmerkungen füllte, die die Einzelheiten und die Ausnahmen, d.h. die gelebten Umstände erläuterten. Es galt also zunächst, diesen Strang zu nutzen, d. h. diese verstreuten Anmerkungen, Andeutungen und diese kurzen Ausblicke auf Hintergründe möglichst vollständig zu erfassen, die in der Masse der uns vorliegenden historischen, literarischen, inschriftlichen oder juristischen Texte auf uns gekommen sind. Es ging also um eine erneuerte Lektüre der häufig sehr bekannten und manchmal weniger vertrauten Texte unter diesem besonderen und ziemlich neuen Gesichtspunkt. Viele Untersuchungen, die Teilaspekte meines Themas behandelten, haben mir natürlich die Aufgabe erheblich erleichtert. Um nur einige Beispiele zu nennen, will ich die Arbeiten von Emilio Gabba, von Peter A. Brunt oder Jacques Harmand zum römischen Heer erwähnen, oder die der Gruppe, die man die amerikanische Schule nennen könnte (von George W. Botsford bis Lily Ross Taylor), zur Organisation und dem Ablauf der "Versammlungen, wo man wählte«. Die Fragen zum Fiskus hatten indes bei weniger Historikern Interesse gefunden und in diesem Bereich musste ich fast bei null anfangen. Am Ende hat sich die mit Hilfe meiner Vorgänger oder ohne sie erkundete Masse der Quellen, die uns die Antike überliefert hat, als ertragreicher herausgestellt als angenommen. Die gesuchten Zeugnisse, die schon bei den Historikern selbst, angefangen mit Polybios und Titus Livius, ziemlich häufig zu finden waren, sind vor allem in den Texten zahlreich, die von ihrer Anlage her dem täglichen Leben näher standen. Für das Theater und die satirische Dichtung war das einigermaßen normal. Dann gab es da die Grammatiker oder die Lexika oder einige bevorzugte Werke, denen der Autor geradezu eine Inszenierung in journalistischem Stil gab (ich denke in dieser Hinsicht besonders an die bemerkenswerten Res Rusticae des Varro). Ebenfalls ist die rhetorische, juristische oder politische Literatur zu nennen, die Einzelne oder bestimmte Gruppen in Szene setzen. Und schließlich ist da jene zwar nicht erschöpfende und in vieler Hinsicht verschlüsselte, aber doch unersetzliche Quelle der Briefe Ciceros. Es ist nützlich, so von Anfang an zu präzisieren, wie meine Dokumentation sich zusammensetzt, und es würde sich lohnen, in forschungstheoretischer Weise zu fragen, welchen Wert sie als Muster darstellt (was ich ohne Frage an anderer Stelle tun werde). Der besorgte Leser kann wenigstens sicher sein, während er mich hier liest, dass ich mir die Frage fortwährend gestellt habe. Mein erster Schritt war also eine einfache neuerliche Lektüre der von den Historikern des republikanischen Roms üblicherweise benutzten Quellen, die indes neuen Einsichten Aufmerksamkeit schenkte [...]. Die Mühe, die übrigens durchaus erfreulich war, wird 
nicht vergeblich gewesen sein, wie ich hoffe. Ich kann sogar sagen (und ich werde mich bemühen, das an anderer Stelle unter Beweis zu stellen), dass sie durch die einfache Aufreihung der Beurteilungen, die Andeutungen und die lediglich an der einen oder anderen Stelle anerkannten Tatsachen erlaubt hat, eine beträchtliche Zahl von traditionellen Irrtümern bei der Interpretation richtigzustellen, zu einigen Punkten ein besseres Verständnis zu eröffnen und ganz einfach manchen berühmten Text besser zu übersetzen. Diese bescheidenen Fortschritte sind in meinen Augen der größte Lohn dieser Arbeit. Man durfte sich natürlich nicht allein auf die Texte beschränken. Eine meiner ständigen Sorgen war es, wie ich schon hervorgehoben habe, die Aktivitäten und unterschiedlichen Rollen, die ihre Eigenschaft als Bürger den jeweiligen Personen auferlegte, in ihren geographischen und zeitlichen Raum einzuordnen. Auf den Spuren von Zvi Yavetz oder Lily Ross Taylor, aber unter Ausdehnung meiner Untersuchung auf die militärischen und steuerlichen Angelegenheiten, habe ich versucht, den Bürgern Schritt für Schritt bei ihren Handlungen sowie zuallererst bei ihren Ortsveränderungen zu folgen, die ihr Dasein als Bürger begleiteten. Auffällig ist die Bedeutung des monumentalen Dekors, das die Stadt Rom, ganz wie die griechischen und hellenistischen Städte, sich veranlasst sieht zu errichten, um als Rahmen für dieses Leben der Gemeinschaft zu dienen. Bei jedem Schritt einer Untersuchung zum Thema stößt man auf topographische Probleme. Es reicht nicht allein zu versuchen, die verschiedenen Momente und wichtigsten Punkte dieses Lebens als Bürger örtlich genau festzulegen oder die Entfernungen und die räumliche Ausdehnung zu berechnen. Man muss sich auch über die gegenseitige und konstante Beziehung klarwerden, die zwischen diesem topographischen und monumentalen Rahmen (in seiner Beständigkeit oder seinen Veränderungen) und den sich dort vollzogenen Handlungen entsteht: sie beeinflussen sich unablässig gegenseitig. Bisweilen bestimmen der Rahmen und die materiellen Einrichtungen die Gestaltung und am Ende auch die Bedeutung einer gewissen Formalität, bisweilen erfordert im Gegenteil die Veränderung der Institutionen die Umgestaltung des Dekors. Nichts ist kennzeichnender, um dieses permanente Wechselspiel von Form und Hintergrund $\mathrm{zu}$ illustrieren, als die Betrachtung der Wahlvorgänge und der technischen Ausgestaltung, die sie voraussetzen.

Nun haben unsere Kenntnisse in diesem Bereich seit einigen Jahren wahrhaft bedeutende quantitative Fortschritte gemacht. Die Topographie Roms zur Zeit der Republik ist schrittweise immer deutlicher geworden, und das dank der Bemühungen italienischer und ausländischer Archäologen und vor allem seit der nahezu definitiven Publikation der berühmten Fragmente des marmornen Plans der Forma Urbis der severischen Zeit, der seit langem bekannt war, aber erst seit kurzem genutzt werden kann. Durch einen glücklichen Zufall ist die Zone, die am meisten von diesen neueren Fortschritten profitiert hat, eben die, in der sich die meisten der Zeremonien des Lebens der Bürger vollzogen, d.h. das Marsfeld. Die Abhaltung des Zensus, das Aufgebot und das Exerzieren des Heeres, die meisten Versammlungen zu Wahlen und Gesetzgebung und schließlich auch die Lagerung und die Verteilung des öffentlichen Getreides fanden in verschiedenen abgegrenzten Bezirken in dieser Zone Roms statt. Natürlich sind bei weitem noch nicht alle Probleme gelöst und es bestehen weiter Unsicherheiten, auch wenn die wesentlichen Fragen geklärt sind.

Ich musste daher auch von dieser Seite her Untersuchungen anstellen, bisweilen mit der Genugtuung, in der Lage zu sein, den professionellen Archäologen und 
Topographen ein Detail zu bestätigen. Umgekehrt ist es häufiger die Prüfung der Topographie, die helfen kann, Probleme der Prozedur zu klären. Ich möchte an dieser Stelle Filippo Coarelli danken, dem großartigen Kenner der Topographie Roms in republikanischer Zeit, der gewillt war, diese Fragen während einer Reise nach Rom im Jahre 1975 ausführlich mit mir zu erörtern.

\section{Eine Fragestellung}

16 Wenn damit das Thema und die Methode in ihren großen Linien definiert waren, musste man noch entscheiden, welche Art von Argumentation man führen wollte. Man musste im Fortschreiten der Arbeit die vorgefassten Vorstellungen oder die bei einem solchen Vorhaben unvermeidlichen Arbeitshypothesen in Frage stellen. Die Geschichte, die ich zu schreiben versuchte, war mit Sicherheit keine politische Geschichte im traditionellen Sinne des Wortes, da sich doch die Politik im engeren Sinne (die Debatten, die Entscheidungen des Vorgehens, die Auseinandersetzungen um die Ausübung der Macht) in Rom, wie man gesehen hat, in einer hierarchischen und sozialen Sphäre abspielte, zu der die einfachen Bürger kaum Zugang hatten. Es handelte sich auch weder um Rechtsgeschichte noch um die Geschichte der Institutionen (obwohl beide mir unentbehrlich waren), da einer der Punkte, die ich erforschen wollte, doch gerade die Distanz und die Beziehungen zwischen Recht und tatsächlichem Geschehen war, zwischen der Regel und der Lebenswirklichkeit. Die Politische Anthropologie und die Politischen Wissenschaften boten mir vielleicht einen Ausweg, doch nicht ohne die notwendigen Reserven. Diese beiden Disziplinen mit ihren zahllosen Varianten beschäftigen sich stets mehr oder minder mit den Beziehungen zwischen Macht und Gesellschaft im ersten Fall, den Regeln und dem Funktionieren des Staates im anderen. All diese Fragen mussten mir ohne Zweifel stets gegenwärtig sein, aber ich musste sie doch nicht direkt als solche behandeln. Zum Beispiel hatte ich mich dagegen entschieden, Fragen zu stellen über den nach einem vorangehenden Stadium erfolgten Aufstieg zu jener besonderen Form der politischen Organisation, die eine antike Stadt darstellt, sondern nur solche über die Bedeutung der Zugehörigkeit zu einer bereits bestehenden und für die ihr angehörenden Bürger voll entwickelten Stadt. Ich musste mir auch nicht nach Art der englischen Schule Fragen über die verschiedenen »Funktionen« stellen, die die Politik oder das Leben als Bürger in der Gesamtheit der sozialen Beziehungen einnehmen konnte, auch wenn dies ein faszinierendes Thema ist und in Hinblick auf Rom noch nicht häufig angeschnitten wurde. Andererseits war es nicht die Erforschung der Strukturen des römischen Staats, der ich mich widmen musste, denn das, was ich als Leben der Bürger definiert hatte, ging weit über das enge Instrumentarium der staatlichen Organisation hinaus. Politische Anthropologie und Politische Wissenschaften sollten mir demnach (da ich übrigens in diesen Disziplinen ja nur ein Autodidakt bin) sehr nützliche Vergleichsmöglichkeiten, Vorstellungen oder, wie man sagt, anregende »Topoi« bieten Eine systematische Übernahme ihrer Methoden wäre nicht dienlich gewesen.

Wenigstens gilt das, wenn man nicht auf die Quellen zurückgehen will. Wenn Rom eine Stadt nach griechischem oder mediterranem Vorbild ist, dann muss man vielleicht den wahren "Gründervater« dieser Disziplinen im Detail befragen. Damit meine ich natürlich Aristoteles, und man sollte sich mit Bedacht in die ihm eigene Vorstellungswelt versetzen, um den Versuch einer ersten Erkundung der 
Charakterzüge der Stadt Rom zu unternehmen. Dabei ging es nicht etwa darum, nach so vielen anderen einen neuen Anlauf zur schwierigen, aber aufschlussreichen Auslegung der Politik zu nehmen. Aber es schien mir nach vorangehenden Untersuchungen doch deutlich zu sein, dass die Erklärungen, die die hellenisierten Römer am Ende der Republik oder die an Rom interessierten Griechen (von Polybios bis zu Dionys von Halikarnass) von den Ursprüngen und den Strukturen der Stadt gaben, sich - sei es unüberlegt oder wohlbegründet - in den Rahmen der aristotelischen Typologie der Städte einfügten. Warum sollte man eigentlich Leuten nicht glauben, die bessere Experten sind als ich? Die Definition der Stadt als einer "societas" (koinonia), einer auf eine stillschweigende Übereinkunft gegründeten Gesellschaft (Politik 1276b) unter Einschluss der allgemeinen und elementaren Definition des Bürgers als dem, dem eine Teilnahme an den richterlichen Funktionen und den Ämtern zusteht (1275a22), wird von den Römern des 2. Jahrhunderts v. Chr. stillschweigend und im 1. Jahrhundert v. Chr. von Cicero ausdrücklich als selbstverständlich übernommen. Die aristotelische Behandlung der »Teile« (merè 1328b) des gemeinschaftlichen Lebens, die zum Überleben der Stadt notwendig sind, und zwar in der aufsteigenden Folge ihrer Bedeutung die ökonomische Funktion (Ernährung und technische Fertigkeiten), die Waffen, das Münzwesen, der Erfüllung der religiösen Pflichten und schließlich, alles andere übertreffend, das Treffen überlegter Entscheidungen (krisis) in Hinblick auf die gemeinschaftlichen Angelegenheiten und die inneren Konflikte, ist bemerkenswert praxisorientiert. Er liefert damit genauer betrachtet einen Leitfaden, der es erlaubt, sich den konkreten Verlauf des Lebens in der Stadt vorzustellen. Das ist mit anderen Worten ein Gesamtbild des Menschen als Bürger, eine angemessene politische Anthropologie. Gewiss vereinfache ich das in meinem Bemühen um eine Zusammenfassung $\mathrm{zu}$ sehr. Man muss vielmehr diese Anthropologie durch eine besonders aufmerksame Lektüre aus dem corpus der aristotelischen Werke herauslösen, ohne dabei zu vergessen, dass Aristoteles in einem gigantischen Bemühen um eine Synthese versucht hat, der extremen Verschiedenheit der politischen und konstitutionellen Formen der vielen hundert griechischer Städte, deren Inventar und Untersuchung er unternommen hatte, in verschiedenen Stadien ihrer jeweiligen Entwicklung Rechnung zu tragen. Seine Anthropologie entspricht der Ausdehnung und dem Erscheinungsbild der griechischen Welt, und das erklärt unter anderem, warum es sich um eine Anthropologie des Wandels und der Bewegung handelt, die nach einem geeigneten »Modell« zur Erklärung der konstanten Anpassungen sucht, die sich vor seinen Augen vollzogen. Als sie sich vornahmen, die römischen Realitäten an dieser Elle zu messen, sahen sich die Autoren, von denen wir ausgehen müssen (Polybios, Cicero, Dionys von Halikarnass) vor einem scheinbar geringeren Problem, weil es sich um nur eine Stadt handelt, deren historische Entwicklung wir doch schließlich besser kennen (oder zu kennen meinen) als die einer jeden griechischen Stadt. Das war eine Stadt von gewaltiger Größe (und bereits an diesem Punkt spüren wir einen wesentlichen Unterschied), deren Konstitution aber relativ stabil und konservativ bleibt und die regionalen Unterschiede Italiens unter dem einheitlichen Überbau des Rechts der Quiriten zusammengehalten hat. Das war eine Stadt, die Polybios und nach ihm im Großen und Ganzen auch Cicero und Dionys von Halikarnass in die Klasse der »moderaten Oligarchien" eingeordnet haben, welche in der Tat der idealen "Mischverfassung" sehr nahestanden, die Aristoteles nach einer langen Erörterung zu der am wenigsten schlechten der Regierungsformen erklärte. 
18 Nun sind innerhalb dieser Verfassung, die die jeweiligen Beziehungen zwischen Volk, Beamten und dem Senat (die Gegenstand der berühmten Analyse des Polybios im 6. Buch seines Werks sind) empirisch bestimmt, die Regeln der Beteiligung aller Bürger an den wesentlichen gemeinschaftlichen Aktivitäten, d. h. der Zugehörigkeit zu dieser speziellen "Gemeinschaft«, die die Stadt darstellt, sowohl von Dionys von Halikarnass wie von Cicero besonders deutlich festgehalten und ausführlich erörtert worden. Am Ende einer so breit wie möglich angelegten Untersuchung erwartet uns die angenehme Überraschung festzustellen, dass die logischen Verbindungen, von denen wir uns vorgestellt hatten, sie unter Beweis zu stellen, d. h. mit anderen Worten die "Struktur«, die uns als grundlegend und zentral erschienen war, bereits von einigen antiken Autoren klar zum Ausdruck gebracht worden waren. Genauer gesagt hatten sie mithilfe des machtvollen Rüstzeugs des griechischen philosophischen Denkens das Wesentliche vom Anekdotischen zu trennen versucht. Dionys von Halikarnass und Cicero, wie nach ihnen Livius und vielleicht vor ihnen Polybios, versichern mit Nachdruck, dass nach ausführlicher Betrachtung die gesamte Stadt Rom auf einer einzigen Institution beruhe, die in der Vielfältigkeit ihrer Folgen grundlegend sei, und das sei der census. Bürger sein heißt, Teil einer Gemeinschaft zu sein, die, wie Aristoteles es ausdrückte, auf einem stillschweigenden Vertrag beruhte. Diese Gemeinschaft setzte die Beteiligung aller ihrer "Angehörigen« an den Aufgaben wie an den Vorteilen voraus. Das einzige Problem ist $\mathrm{zu}$ entscheiden, aufgrund welchen Prinzips sich diese Beteiligung vollzog. Aristoteles hatte bereits zutreffend vorausgeahnt, dass die Revolutionen, die Erfolge und die Niederlagen der Städte zu einem großen Teil von der Kraft des consensus abhingen, der die Bürger miteinander verband oder auch nicht. In den Augen aller unserer Autoren erscheint der consensus, der die Stadt Rom beseelte, zumindest bis hin zum beginnenden 1. Jahrhundert v. Chr., als besonders stark und widerstandsfähig gegenüber allen inneren Spannungen wie angesichts der schrecklichsten Herausforderungen, die von außen kamen. Das erklärt sich in ihren Augen aus der herausragenden Qualität des Prinzips, das die Teilnahme an den verschiedenen Aktivitäten der Bürgerschaft regelt und die Vorteile und Nachteile des gemeinschaftlichen Lebens innerhalb der Gesellschaft verteilt. Ausnahmsweise bestätigen ganz eindeutige Texte, die man natürlich jeden für sich in den Zusammenhang eines Denkens und einer Kultur einordnen muss (aber das habe ich an anderer Stelle getan), in fast vollständig entsprechender Weise die empirischen und konkreten Zeugnisse, die ich in diesem Buch zusammengetragen und geordnet habe. Es wäre ziemlich übertrieben, wie man zugeben wird, diese bequeme Methode zurückzuweisen: auf der Ebene der Darstellung liefert sie einen logischen Rahmen, der für uns wenigstens den Vorteil hat, Anachronismen $\mathrm{zu}$ vermeiden. Wir wollen Aristoteles, Dionys und Cicero zusprechen, was ihnen gebührt. Sie sind sicherlich nicht die einzigen, denen ich Begriffe, Konzepte oder »Topoi« entlehnt habe, aber es sei mir doch erlaubt, meine Schuld ihnen gegenüber zu erklären. Wir werden am Ende sehen, dass man natürlich auch eine andere »Lektüre» des politischen Systems Roms umreißen kann, dessen Grundlagen ich habe erkunden wollen. Man könnte etwa, um die Blickrichtung zu verändern, cum grano salis von Claude Lévi-Strauss oder David Easton eine gewisse in Mode gekommene Sprache entlehnen und von »Struktur « oder "System» sprechen. Aber nichts reicht bis jetzt an Polybios, Dionys von Halikarnass oder Cicero heran, um Rom zu verstehen.

Das Gesagte erklärt einigermaßen klar, wie ich meine, den Inhalt dieses Buchs. Ich habe das Leben der Bürger in den in meinen Augen wesentlichen drei Dimensionen 
untersucht: dem militärischen Bereich, dem der Steuern und Finanzen und schließlich dem der Beratung und Wahl. In allen drei Bereichen habe ich versucht, den grundlegenden Einfluss der Organisation des Zensus und dessen Prinzipien und Konsequenzen hervorzuheben. Aber es schien mir doch so zu sein, dass die harmonische Sprache, die im 3. und 2. Jahrhundert v. Chr. die Regeln der Beteiligung in diesen drei Bereichen festlegte, aus verschiedenen Gründen, die ich vorgebracht habe, am Ende des 2.Jahrhunderts v. Chr. zu verfallen begann. Offenbar genügte das Wechselspiel der Institutionen des Zensus nicht mehr, um auf alle Bedürfnisse des politischen und sozialen Verbunds nach Kommunikation eine Antwort zu geben. Die drei Säulen des republikanischen Staats (das Bürgerheer, der steuerliche Wetteifer, die Disziplin bei den Wahlen) verschwanden in logischer Folge eine nach der anderen. Infolgedessen entstanden neue Wege der Kommunikation oder zur Erreichung von consensus sowie neue Kommandostränge, und es scheint mir, dass man neben dem alten Sprachgebrauch und den alten Begriffen bürgerlichen Verhaltens eine neue Begrifflichkeit und eine neue Sprache fand. Ich habe versucht, das in kürzerer Form zu untersuchen, und zwar in erster Linie deshalb, weil das Thema neu ist und noch viele Forschungen und Untersuchungen erfordert.

Es ist mir dennoch unerlässlich erschienen, den Vorgang in diesem Buch zu umreißen, selbst auf die Gefahr hin, das Bild des Lebens der Bürger im letzten Jahrhundert der Republik gefährlich zu verfälschen.

Der Leser wird beunruhigt feststellen, dass ein besonders wichtiges Thema fehlt: die Religion. Die religiöse Dimension der Angelegenheiten der Bürger ist mir indes als aufmerksamem Leser Fustel de Coulanges nicht etwa entgangen. Doch das Thema, das von unseren Quellen und der heutigen Forschung bevorzugt behandelt wird und als solches so gewaltig ist, steht meinen Neigungen und meinen Kompetenzen fern. Es ist besser, das unumwunden zuzugeben. Hingegen zieht das Thema, das ich für meine Abhandlung gewählt habe, eine notwendige Erweiterung nach sich. Ich meine das Studium der offiziellen Gruppierungen der Bürger, denen sie nicht als Bürger angehören: Vereine und Kollegien aller Art oder - vielleicht und unter Bedingung einer Bestandsaufnahme - sogar politische »Parteien«. Diese alten oder neuen Strukturen formen das bürgerliche Verhalten nicht weniger deutlich als die Zugehörigkeit zu einer Tribus oder die Teilnahme an der Volksversammlung. Ich bin mir dessen sehr wohl bewusst. Aber es fehlte mir an Platz. Ich habe ihre umfassende Untersuchung für einen späteren Zeitpunkt vorgesehen.

\section{BIBLIOGRAPHIE}

Badian, E. (1958): Foreign Clientelae 264-70 (BC), Oxford.

Dumont, Louis M. (1976 [1966]): Gesellschaft in Indien. Die Soziologie des Kastenwesens übers. von Margarte Venjakob, Wien.

Garnsey, P. (1970): Social Status and Legal Privilege in the Roman Empire, Oxford. 
Gruen, E. (1968): Roman Politics and the Criminal Courts 149-73 B.C., Cambridge / Mass.

Gruen, E. (1974): The Last Generation of the Roman Republic, Berkeley.

Hassal, M. /Crawford, M. / Reynolds, J. (1974): »Rome and the Eastern Provinces at the End of the Second Century B.C.«, JRS 64, S. 195-220.

Kelly, J. (1966): Roman Litigation, Oxford.

Münzer, F. (1920): Römische Adelsparteien und Adelsfamilien, Stuttgart.

Nicolet, C. (1970): »Prosopographie et histoire sociale: Rome et l'Italie à l'époque républicaine«, Annales E.S.C., S. 1209-1228.

Nicolet, C. (1972): „Les lois judiciaires et les tribunaux de concussion. Travaux récents et directions de recherches«, in: Aufstieg und Niedergang der Römischen Welt (ANRW), Bd. I.2, S. 197214.

Scullard, H. H. (1951): Roman Politics 220-150 B.C., Oxford.

Syme, R. (1939): The Roman Revolution, Oxford.

\section{NOTES}

1. Syme (1939), S. 7.

2. Das ist das berühmte "Gesetz von Delphi«, das ein Kommando gegen die Piraten schafft (Fouilles de Delphes III, nr. 37 = FIRA nr. 9); eine neue Version dieses Textes ist in Knidos entdeckt worden (Hassall / Crawford / Reynolds [1974]).

3. Der Ausdruck ist natürlich nicht wirklich geeignet; es gibt keine »Gewalt« der Rechtsprechung (vgl. meine Bemerkungen in ANRW, Bd. I,2 (= Nicolet [1972]), und das grundlegende Werk von Gruen [1968]).

4. Im Bereich der politischen Geschichte hat die Prosopographie als eine berechtigte Reaktion auf den »Konstitutionalismus« der Schule Mommsens unsere Begriffe von Strukturen und Konflikten vollständig erneuert (vgl. Nicolet [1970], S. 1214-1216). Den Wendepunkt markiert Münzer (1920); die Methode ist seitdem systematisch eingesetzt und von denen, die man die angelsächsische Schule nennen könnte, entwickelt worden: Syme (1939); Scullard (1951); Badian (1958); mit Gruen (1974) scheint sie an ihre Grenzen gestoßen zu sein.

5. $\mathrm{Zu}$ dem Abstand, den wir in Hinblick auf bestimmte Gesellschaften eine Typs gewinnen müssen, die sich von der unseren radikal unterscheiden, s. Dumont (1976 [1966]), S. 17-38. Das römische Zivilrecht ist während einer bestimmten Zeit einer egalitären Tendenz gefolgt (aequum ius: Cic. rep. 1,49). Aber weder das Strafrecht noch das Staatsrecht oder selbst die reale Praxis des Privatrechts waren egalitär; vgl. Garnsey (1970); Kelly (1966).

\section{INDEX}

Mots-clés : République romaine, citoyen, citoyenneté

Schlüsselwörter : römische Republik, Bürger, Bürgerschaft 


\section{AUTEURS}

\section{CLAUDE NICOLET}

Claude Nicolet (1930-2010) war einer der wichtigsten französischen Althistoriker. Nähere

Informationen finden Sie hier. 